\title{
Hepatotoxicidade grave secundária a psicofármacos e indicação de eletroconvulsoterapia a paciente com esquizofrenia
}

\author{
Severe psychotropic-induced hepatotoxicity and indication of electroconvulsive \\ therapy in a patient with schizophrenia
}

\author{
Maila de Castro Neves, Arthur Kummer, Luciana Monteiro Luciano, Hélio Lauar, João \\ Vinícius Salgado, Rodrigo Nicolato e Antônio Lúcio Teixeira
}

\section{Resumo}

Relatamos o caso de uma paciente com diagnóstico de esquizofrenia paranóide que desenvolveu dois quadros de hepatotoxicidade atribuídos a efeitos colaterais do ácido valpróico e da clozapina. Essa relação foi estabelecida pela proximidade temporal entre o início da medicação e a instalação do quadro, pela resolução deste após a suspensão da medicação e pela exclusão de outras causas de doença hepática. No seguimento, o controle clínico da paciente foi realizado com eletroconvulsoterapia (ECT). Salientamos a importância da vigilância cuidadosa dos efeitos adversos de psicofármacos, especialmente sobre a função hepática, e da ECT como possibilidade terapêutica diante de limitações farmacológicas.

Palavras-chave: hepatotoxicidade, ácido valpróico, clozapina, eletroconvulsoterapia.

\begin{abstract}
We report the case of a patient with paranoid schizophrenia who developed two episodes of severe hepatotoxicity. They were attributed to the side effects of valproic acid and clozapine. This relationship was established by the temporal proximity between the onset of medication and the development of side effects, the clinical remission after drug cessation and the exclusion of other causes of hepatic diseases. On the follow-up, the patient was treated with eletroconvulsive therapy. This case report highlights the relevance of careful vigilance of adverse effects of psychotropics, especially hepatotoxicity, and the role of eletroconvulsive therapy when there are pharmacological constraints.

Key words: hepatotoxicity, valproic acid, clozapine, eletroconvulsive therapy.
\end{abstract}

Hospital de Ensino Instituto Raul Soares (IRS), Fundação Hospitalar de Minas Gerais (FHEMIG) (Neves MC, Kummer A, Lauar H, Salgado JV, Nicolato R)

Recebido 16-11-05 Aprovado 15-03-06

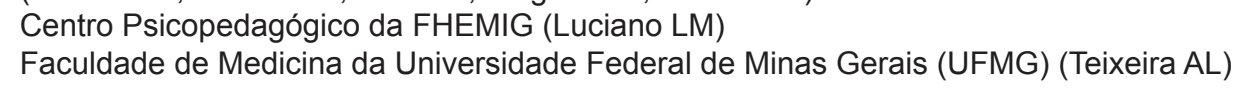

Correspondência para: Antônio Lúcio Teixeira

Departamento de Clínica Médica, Faculdade de Medicina da UFMG - Av. Prof. Alfredo Balena, 190 - 30130-100

- Belo Horizonte-MG - Telefax: (31) 3499-2651 - e-mail: altexr@gmail.com 


\section{Introdução}

A lista de fármacos capazes de provocar efeitos adversos hepáticos inclui mais de mil medicamentos, dos quais cerca de $15 \%$ são neuropsiquiátricos (Aubriot-Delmas, 1997). Nos Estados Unidos, em 2003, mais de $75 \%$ dos casos de reação idiossincrásica hepática a drogas resultaram em transplante hepático ou morte, o que ilustra a gravidade potencial de tais efeitos (Lee, 2003).

Relatamos o caso de uma paciente de 39 anos, com diagnóstico de esquizofrenia paranóide, que desenvolveu dois quadros de hepatotoxicidade grave com o uso de ácido valpróico (VPA) e de clozapina. Salientamos a importância da vigilância cuidadosa dos efeitos colaterais de drogas psicotrópicas, especialmente sobre a função hepática, dos fatores de risco para tais efeitos, além das possibilidades terapêuticas da eletroconvulsoterapia (ECT) diante de restrições ao uso de psicofármacos.

\section{Relato de caso}

Trata-se de uma mulher de 39 anos, solteira, sem filhos, do lar, com seis anos de escolaridade formal, católica. Iniciou acompanhamento psiquiátrico no Instituto Raul Soares (IRS) em 1988, aos 23 anos, com diagnóstico de esquizofrenia paranóide, apresentando, na época, alterações da consciência do eu, delírios persecutórios, alucinações auditivas, visuais e cenestésicas. Além disso, seu comportamento era marcado por erotização, agressividade, errância e negligência em relação ao cuidado pessoal. A história médica pregressa da paciente, assim como a familiar, não apresentava eventos ou alterações dignas de nota. A paciente também negava uso anterior de bebidas alcoólicas ou outras substâncias psicoativas.

De 1988 até 2001, a paciente fez uso de haloperidol até $25 \mathrm{mg} / \mathrm{dia}$ e tioridazina até $500 \mathrm{mg} / \mathrm{dia}$, sem resposta clínica satisfatória. Em 2001, após iniciar uso de olanzapina (até 30mg/ dia), evoluiu com leve melhora na capacidade de autocuidado, embora mantivesse sintomas delirantes e alucinatórios proeminentes. No início de 2004, a atividade delirante persecutória acentuou-se com conseqüente aumento da hostilidade. Foi associado VPA $500 \mathrm{mg} / \mathrm{dia}$ à olanzapina, então na dose de $20 \mathrm{mg} / \mathrm{dia}$. Após quatro semanas, a paciente passou a exibir estado confusional hipoativo, caracterizado por rebaixamento do nível de consciência, prostração, apatia e mutismo. Ao exame físico, mostrava-se eutrófica, hidratada, normocorada, mas ictérica. Encontrava-se com níveis tensionais de $120 / 80 \mathrm{mmHg}$, freqüência cardíaca de $76 \mathrm{bpm}$, eupnéica, sem ruídos adventícios à ausculta pulmonar, abdome normotenso, dolorido difusamente, sem massas palpáveis. Foi internada, então, em hospital geral para a investigação clinicolaboratorial do possível quadro de doença hepática aguda. Os exames laboratoriais revelaram aumento das bilirrubinas total $(6,4 \mathrm{mg} / \mathrm{dl})$ e direta $(5,4 \mathrm{mg} / \mathrm{dl})$, das enzimas hepáticas (transaminase glutâmico-pirúvica [TGP] = 242U/I, transaminase glutâmico-oxalacética [TGO]= 112U//, gama-glutamil transpeptidase $[\mathrm{GGT}]=100 \mathrm{U} / \mathrm{I}, \mathrm{FA}=75 \mathrm{U} / \mathrm{I}$ ) e do tempo de protrombina (razão normalizada internacional $[R N I]=2,4)$. O restante dos exames laboratoriais, incluindo hemograma, ionograma, função renal, glicemia, amilase e lipase pancreáticas, não mostrou alterações. 0 exame de ultra-sonografia abdominal evidenciou leve aumento difuso do fígado. Como as sorologias para as hepatites virais $\mathrm{B}$ e $\mathrm{C}$ foram negativas, assim como foi descartado o diagnóstico de doença de Wilson, estabeleceram-se, então, os diagnósticos de encefalopatia hepática e hepatite medicamentosa possivelmente secundária ao VPA. A medicação psiquiátrica foi suspensa, mas a paciente permaneceu com redução do nível de consciência por cerca de 20 dias. Posteriormente houve melhora clínica progressiva e normalização das provas de função hepática com recuperação completa em cinco semanas.

A paciente retornou ao ambulatório do IRS com comportamento bastante desorganizado e sintomas psicóticos proeminentes, o que motivou a reintrodução do haloperidol $10 \mathrm{mg} / \mathrm{dia}$. Entretanto não apresentou boa resposta terapêutica, evoluindo com significativas alterações comportamentais, como mutismo, negativismo, apatia e negligência pessoal. Diante da precariedade da resposta clínica aos diferentes antipsicóticos empregados, optou-se por interná-la no IRS e iniciar o uso de clozapina. As revisões clínica e laboratorial não evidenciaram contra-indicações para a introdução desta medicação, sendo que o hemograma e as provas de função hepática eram repetidos semanalmente. Quando estava em uso de $150 \mathrm{mg} / \mathrm{dia}$ de clozapina, seis semanas após o início da medicação, a paciente passou a apresentar períodos de oscilação do nível de consciência, prostração, apatia e febre de $39^{\circ} \mathrm{C}$. Os exames laboratoriais revelaram aumento das enzimas hepáticas $(T G P=470 \mathrm{U} / \mathrm{l}, \mathrm{TGO}=88 \mathrm{U} / \mathrm{l}, \mathrm{GGT}=195 \mathrm{U} / \mathrm{l}$, fosfatase alcalina $[F A]=84 U / I$ ) e neutropenia (leucócitos totais: $5.100 \mathrm{~mm}^{3}$, neutrófilos $3 \%$ e linfócitos $78 \%$ ), sem outras alterações. Foi encaminhada novamente ao hospital geral para tratamento de neutropenia febril com antibióticos de largo espectro por 14 dias, embora não tivesse sido identificado foco infeccioso. A hemocultura também não revelou crescimento bacteriano. As alterações das provas de função hepática se normalizaram cerca de sete dias após a suspensão da clozapina.

Após a alta da unidade clínica, em virtude de a paciente mostrar-se bastante desorganizada, agressiva e com sintomas psicóticos acentuados, optou-se por tratamento com eletroconvulsoterapia (ECT), após consentimento da família. Enquanto estava internada no IRS, foram realizadas oito sessões com aplicações bilaterais, utilizando-se aparelho que gera pulsos sinusoidais. Diante da necessidade do uso de fármacos para a anestesia geral no procedimento de ECT, e do conseqüente risco de novo quadro de hepatotoxicidade, a função hepática foi regularmente avaliada. Pelo mesmo motivo foi efetuada apenas uma sessão de ECT por semana. Após quatro sessões, evidenciou-se melhora significativa da desorganização comportamental. Nas sessões seguintes, houve atenuação da hostilidade e da atividade delirante-alucinatória. Em nenhum momento houve elevação das provas de função hepática.

A paciente recebeu alta hospitalar após a oitava sessão de ECT. Seis meses após a alta, em uso de $5 \mathrm{mg} /$ dia de haloperidol, comparece regularmente às consultas ambulatoriais às sessões mensais de ECT. No momento, a paciente não relata alucinações, não apresenta agressividade ou desorganização do comportamento e mantém atividade delirante que não implica risco para si ou terceiros. Apresenta cuidado pessoal 
preservado, tem desenvolvido atividades artesanais e ajudado a família nos afazeres diários.

\section{Discussão}

O presente relato apresenta dois quadros graves sugestivos de encefalopatia hepática que podem ser atribuídos a efeitos colaterais das medicações em uso. Os fatos que permitem estabelecer essa relação de causalidade incluem a proximidade temporal entre a introdução da droga e o aparecimento do problema clínico, a resolução do quadro após a suspensão da medicação e a exclusão de outras causas de doença hepática.

O primeiro quadro de hepatotoxicidade parece ter tido relação com o uso do VPA. A hepatotoxicidade pelo VPA é caracterizada por grau variável de necrose dos hepatócitos, com período de latência entre cinco e 90 dias, sendo que a biópsia hepática raramente é útil para o diagnóstico (Lee, 2003). A hipótese fisiopatológica mais consistente é a de que - VPA sobrecarrega a função mitocondrial, especialmente a capacidade de beta-oxidação e a cadeia enzimática da respiração, determinando acúmulo de produtos tóxicos do metabolismo endógeno e conseqüente morte hepatocelular (Lee, 2003; Silva, 2004; Eadie, 2003). Alguns fatores de risco incluem idade inferior a 2 anos; politerapia, especialmente 0 uso concomitante de drogas que induzem o citocromo hepático P450 (Gopaul, 2003); e anormalidades neurológicas e metabólicas (Bauer, 2005). O quadro clínico é potencialmente fatal, embora a literatura registre uma única descrição de falência hepática fulminante associada ao uso de VPA em monoterapia (Bauer, 2005). Como a paciente também estava em uso de olanzapina, esta poderia ter aumentado o risco de hepatotoxicidade relacionada ao ácido valpróico. Ressalta-se, entretanto, que a olanzapina tem baixa afinidade pelos citocromos hepáticos P450, interferindo pouco no metabolismo de outras drogas e sendo considerada segura quanto à hepatotoxicidade (van Kammen e Marden, 2005).

O segundo episódio de hepatotoxicidade foi associado ao uso de clozapina. Ressalta-se que a alteração enzimática hepática com o uso da clozapina é, na maioria das vezes, benigna e reversível, mesmo sem redução de dose (AubriotDelmas, 1997). Em investigação comparando tolerância hepática e neurolépticos, pacientes tratados com clozapina apresentaram maior freqüência de alterações de enzimas hepáticas $(37,5 \%)$ do que aqueles tratados com haloperidol $(16,6 \%)$ (Aubriot-Delmas, 1997). Estima-se a ocorrência de hepatite colestática e hepatite fulminante fatal em, respectivamente, $0,06 \%$ e $0,001 \%$ dos casos em uso de clozapina (MacFarlane, 1998). O mecanismo pelo qual a clozapina induz hepatotoxicidade não está definido (Aubriot-Delmas, 1997). É interessante afirmar que, apesar de existir uma similaridade estrutural entre a olanzapina e a clozapina, essa semelhança parece traduzir-se apenas pela propensão que possuem ambos os agentes à indução de ganho de peso (van Kammen e Marden, 2005). Como comentado anteriormente, a olanzapina é considerada segura do ponto de vista de toxicidade hepática, o que não ocorre com a clozapina (van Kammen e Marden, 2005).
A suscetibilidade de alguns pacientes ao dano hepático induzido por drogas parece ser explicada por alguns fatores: 1) predisposição genética, como falha em mecanismos protetores como a glutationa-redutase ou variantes genéticas de isoenzimas do citocromo P450; 2) fatores adquiridos, como a presença de desnutrição, alcoolismo e uso de indutores microssômicos; 3) suscetibilidade à hepatite auto-imune desencadeada por drogas (Pessayre, 1992). Ainda não foram desenvolvidos marcadores bioquímicos ou genéticos que possam ser empregados com segurança na prática clínica para a identificação dos indivíduos com maior risco de desenvolverem hepatopatia por drogas. A única espécie de prevenção possível, no momento, é a farmacovigilância clinicolaboratorial.

Concomitantemente ao segundo episódio de hepatotoxicidade, a paciente desenvolveu febre e neutropenia. A agranulocitose associada à clozapina é maior entre as mulheres, aumenta com a idade e tem incidência acumulada de 0,8\% em 12 meses e de 0,91\% em 18 meses (Alvir, 1993; Jauss, 2000). A febre associada à clozapina é comum, variando sua freqüência de $5 \%$ a $55 \%$ dos casos, conforme a metodologia utilizada no estudo, e geralmente não estando relacionada a síndrome neuroléptica maligna nem a septicemia. Contudo, se a febre ocorre associada a neutropenia, a possibilidade de septicemia deve ser investigada, como aconteceu no caso. É importante salientar que a presença de febre não prediz as alterações hematológicas (Tham, 2002).

Diante das limitações farmacológicas, considerou-se o uso da ECT. As evidências científicas atuais indicam que a ECT de curto prazo, quando combinada com antipsicóticos, pode determinar melhora global em pacientes com esquizofrenia (Tharyan e Adams, 2005). Pode ainda ser usada como terapêutica de primeira escolha quando seu risco é suplantado pelos riscos dos tratamentos convencionais e no caso de intolerância a efeitos colaterais que não podem ser evitados e que se acredita serem menos prováveis ou graves com a ECT (American Psychiatric Association, 2001; Weiner, 1994). A ECT é efetiva para o tratamento de exacerbações psicóticas na esquizofrenia, mesmo em pacientes crônicos, já que existem relatos de resposta em até $20 \%$ dos casos (American Psychiatric Association, 2001; Weiner, 1994). Alguns autores recomendam que a ECT de manutenção deva ser administrada por no mínimo seis meses após a indução da remissão para prevenção de recaídas (Fleck, 1998). Postula-se ainda que o uso combinado de ECT de manutenção com antipsicóticos, para aqueles que responderam a um curso inicial de ECT, é superior ao tratamento de manutenção empregando isoladamente antipsicóticos ou ECT na prevenção de recaídas (Tharyan e Adams, 2005).

Cabe destacar que a ECT ainda é pouco empregada em nosso meio, o que reflete o desconhecimento dos profissionais em relação à eficácia e à segurança dessa terapêutica, assim como as percepções negativas e estigmatizantes sobre a técnica pelo leigo. Comumente o procedimento é postergado em favor de medidas aparentemente menos agressivas, como intervenções farmacológicas, negligenciando o fato de que há riscos consideráveis com o uso de determinadas medicações. 


\section{Conclusão}

O presente relato revela a importância da farmacovigilância como único meio possível de tentar amenizar a gravidade dos efeitos colaterais dos psicofármacos. Estudos são necessários para o desenvolvimento de métodos de prevenção de efeitos colaterais das drogas, como a identificação de pacientes de alto risco a partir de marcadores bioquímicos e/ou genéticos.

\section{Referências}

Alvir JMJ, Lieberman JA, Safferman AZ, Scwimmer JL, Schaaf JA. Clozapine-induced agranulocytosis: incidence and risk factors in the United States. N Engl J Med, 39: 162-7, 1993.

American Psychiatric Association. The practice of ECT: recommendations for treatment, training and privileging. Washington, DC: American Psychiatric Press Inc.; 2001.

Aubriot-Delmas B, Gilkman J, Januel D. Hepatic tolerance of atypical antipsychotic drugs. J Psychopharmacol, 17: 314-7, 1997.

Bauer MS. Fatal hepatic failure and valproate. Am J Psychiatry, 162: 1, 2005.

Eadie MJ, McKinnon GE, Dustan PR. Valproate metabolism during hepatotoxicity associated with the drug. Eur J Intern Med, 14: 33840, 2003.

Fleck M, Schestasky S, Lima AFBS. Desenvolvimento de rotinas para a prática de eletroconvulsoterapia no Hospital das Clínicas de Porto Alegre. J Bras Psiquiatr, 47: 465-8, 1998.

Gopaul S, Frrel K,Abbout F. Effects of age and politherapy risk factors of valproic acid (VPA) hepatotoxicity on the excretion of thiol conjugates of 4-dieneVPA in people with epilepsy taking VPA. Epilepsia, 44: 322-8, 2003.

Jauss M, Pantel J, Werle E, Schroder J. G-CSF Plasma levels in clozapineinduced neutropenia. Biol Psychiatry, 48: 1113-5, 2000.
Van Kammen DP, Marden SR. Serotonin-dopamine antagonists (atypical or second generation antipsychotics). In: Sadock BJ, Sadock VA, editors. Kaplan and Sadock's comprehensive textbook of psychiatry. Lippincot Williams \& Wilkins, p. 2914-38, Philadelphia, 2005.

Lee A. Drug-induced hepatotoxicity. N Engl J Med, 349: 474-85, 2003.

Macfarlane B, Davies S, Mannan K, Sarsam R, Pariente D. Fatal acute and fulminant failure due to clozapine: a case report and review of clozapine-induced hepatotoxicity. Baillieres Clin Gastroenterol, 2: 385-422, 1998.

Pessayre D, Larrey D. Acute and chronic drug-induced hepatitis. Drug Saf, 1: 17-25, 1992

Silva MF, lj|st L, Allers P, Jakobs C, Duran M, de Almeida IT, et al. ValproyldephosphoCoA: a novel metabolite of valproate formed in vitro in rat liver mitochondria. Drug Metab Dispos, 32: 1304-10, 2004.

Tham JC, Dickson RA. Clozapine-induced fever and 1-year clozapine discontinuation rate. J Clin Psychiatry, 63: 10, 2002.

Tharyan P, Adams CE. Electroconvulsive therapy for schizophrenia. The Cochrane Database of Systematic Reviews, 2: CD000076, 2005.

Weiner RD. Treatment optimization with ECT. Psychopharmacology Bull, 30: 313-20, 1994. 